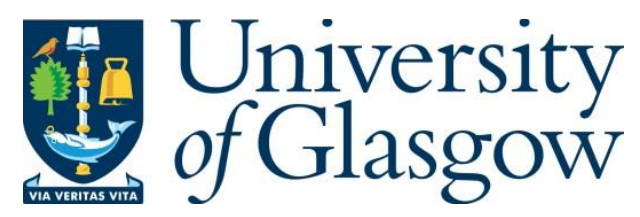

Fischer, M. P. et al. (2017) Mid-Infrared Third-Harmonic Emission from Heavily-Doped Germanium Plasmonic Nanoantennas. In: CLEO: QLES_Fundamental Science 2017, San Jose, CA, USA, 14-19 May 2017, FF1G.2. ISBN 9781943580279.

There may be differences between this version and the published version. You are advised to consult the publisher's version if you wish to cite from it.

http://eprints.gla.ac.uk/141834/

Deposited on: 27 November 2017

Enlighten - Research publications by members of the University of Glasgow http://eprints.gla.ac.uk 


\title{
Mid-Infrared Third-Harmonic Emission from Heavily-Doped Germanium Plasmonic Nanoantennas
}

\author{
Marco P. Fischer ${ }^{1}$, Aaron Riede ${ }^{1}$, Alexander Grupp ${ }^{1}$, Kevin Gallacher ${ }^{2}$, Jacopo Frigerio ${ }^{3}$, \\ Giovanni Pellegrini ${ }^{4}$, Michele Ortolani ${ }^{5}$, Douglas J. Paul ${ }^{2}$, Giovanni Isella ${ }^{3}$, Alfred Leitenstorfer ${ }^{1}$, \\ Paolo Biagioni ${ }^{4}$ and Daniele Brida ${ }^{{ }^{*}}$ \\ ${ }^{1}$ Department of Physics and Center for Applied Photonics, University of Konstanz, 78457 Konstanz, Germany \\ ${ }^{2}$ School of Engineering, University of Glasgow, Rankine Building, Oakfield Avenue, Glasgow, G12 8LT, UK \\ ${ }^{3}$ L-NESS, Dipartimento di Fisica del Politecnico di Milano, Via Anzani 42, 22100 Como, Italy \\ ${ }^{4}$ Dipartimento di Fisica, Politecnico di Milano, Piazza Leonardo da Vinci 32, 20133 Milano, Italy \\ ${ }^{5}$ Department of Physics, Sapienza University of Rome, Rome, 00185 Italy \\ *Author e-mail address: daniele.brida@uni-konstanz.de
}

\begin{abstract}
We investigate the nonlinear optical properties of single resonant plasmonic antennas fabricated from heavily-doped Germanium films. Excitation with intense and ultrashort midinfrared pulses at $10.8 \mu \mathrm{m}$ wavelength produces emission at $3.7 \mu \mathrm{m}$ via third-harmonic generation. OCIS codes: (190.7110) Ultrafast nonlinear optics; (310.6628) Subwavelength structures, nanostructures
\end{abstract}

Recent advances in semiconductor film deposition allow the growth of heavily doped germanium with effective plasma frequencies of up to $60 \mathrm{THz}$, corresponding to $5 \mu \mathrm{m}$ wavelength. This technology paves the way for mid-infrared (MIR) plasmonics with application to integrated telecommunications systems and to precise sensing in the spectral region defined as the vibrational fingerprint of molecules. Characteristics like CMOS compatibility, low electron effective mass and tunable dielectric function give significant advantage to Ge over other semiconductors $[1,2]$. In addition, metals like gold display relatively poor plasmonic performances in the MIR due to a disadvantageous dielectric response that leads to weak confinement of the electromagnetic field.

In this work, we demonstrate that plasmonic Ge antenna structures are also suitable for driving nonlinear optical processes such as third-harmonic generation (THG) in the mid infrared [3] owing to the strong near-field enhancement of the light-matter coupling. These devices act as light emitters constrained at sub-wavelength dimensions and consequently are of high interest for experiments targeting single molecules or other isolated quantum systems at ultrashort timescales [4].

Doped single-crystalline germanium films with a thickness of $1 \mu \mathrm{m}$ are grown epitaxially via low-energy plasma enhanced chemical vapor deposition (LEPECVD) on intrinsic silicon substrates. Annealing steps ensure the highest dopant activation. Resonant antenna structures including double rod antennas (see Fig. 1(a)) are then fabricated via electron beam lithography. Simulations considering the antenna geometry and the material dielectric function close to the plasma edge determine the exact resonance for mid-infrared excitation [5]. The optical characterization system is

(a)
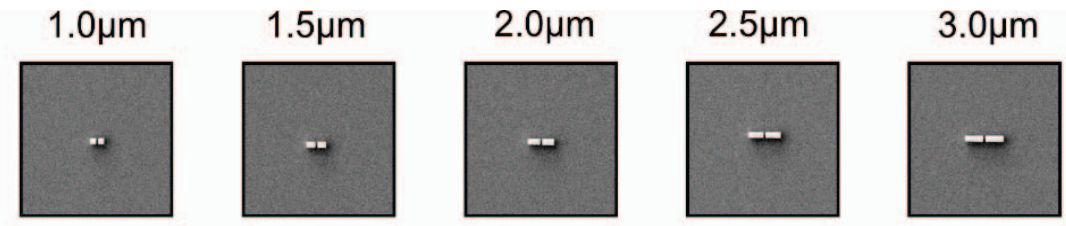

(b)
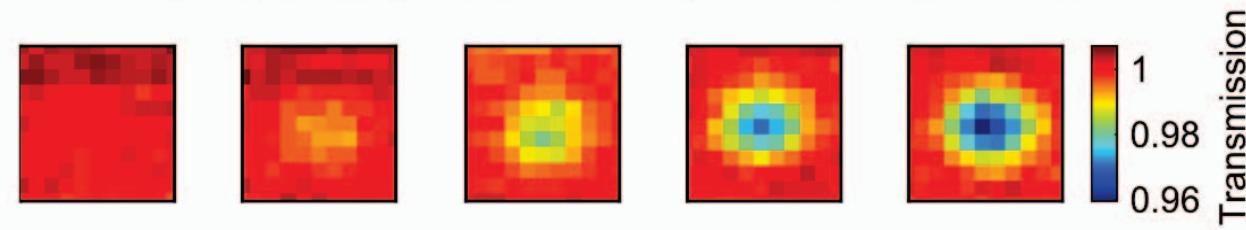

(c)
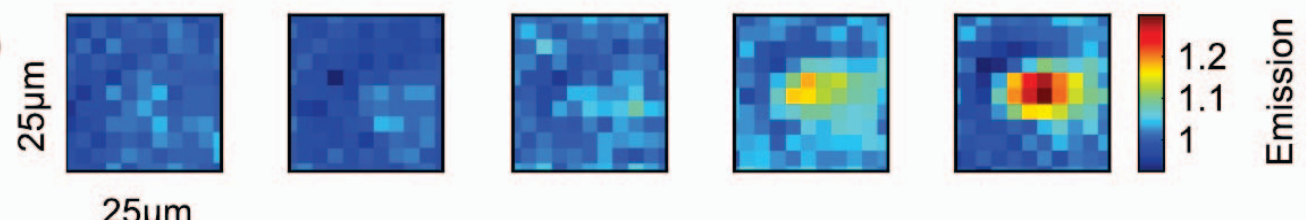

Fig. 1. (a) Scanning electron micrographs of several double rod antennas with increasing arm length; (b) linear transmission maps of each double rod antennas illuminated with a laser pulse centered at the wavelength of $12 \mu \mathrm{m}$; (c) spatially resolved third harmonic emission normalized to the silicon substrate background. The dimension of each panel is $25 \mu \mathrm{m}$ by $25 \mu \mathrm{m}$. 
pumped by a $\mathrm{Yb}: \mathrm{KGW}$ femtosecond laser that drives the nonlinear frequency conversion stages for the generation of intense few-cycle pulses tunable in the mid-infrared spectral range from $8 \mu \mathrm{m}$ to $22 \mu \mathrm{m}$ wavelength. Excitation fields of up to $20 \mathrm{MV} / \mathrm{cm}$ are reached in the focus of home-made microscope equipped with a dispersion-free CassegrainSchwarzschild reflecting objective (NA=0.5). A second, identical objective is used to image the nanostructure in transmission geometry. Dielectric and semiconductor filters as well as a grating monochromator are employed for spectral filtering.

A liquid nitrogen cooled mercury cadmium telluride detector collects the emission while the sample is scanned through the common focus. This setup allows the addressing of single antennas and the linear and nonlinear crosssections to be mapped. We study different antenna geometries, sizes and material doping levels.

Fig. 1(b) demonstrates the transmission images at the fundamental wavelength with the increased scattering of isolated nanostructures while Fig. 1(c) plots the corresponding THG emission that is strongly enhanced at the antenna position with respect to the substrate. Fig. 2(a-b) demonstrates the spectral characterization of the third harmonic photons that are generated at $3.7 \mu \mathrm{m}$ in comparison to the fundamental excitation set at $10.8 \mu \mathrm{m}$.

The experimental variation of the excitation power reveals the nonlinear dependence (Fig. 2(c)) typical for thirdorder optical processes.
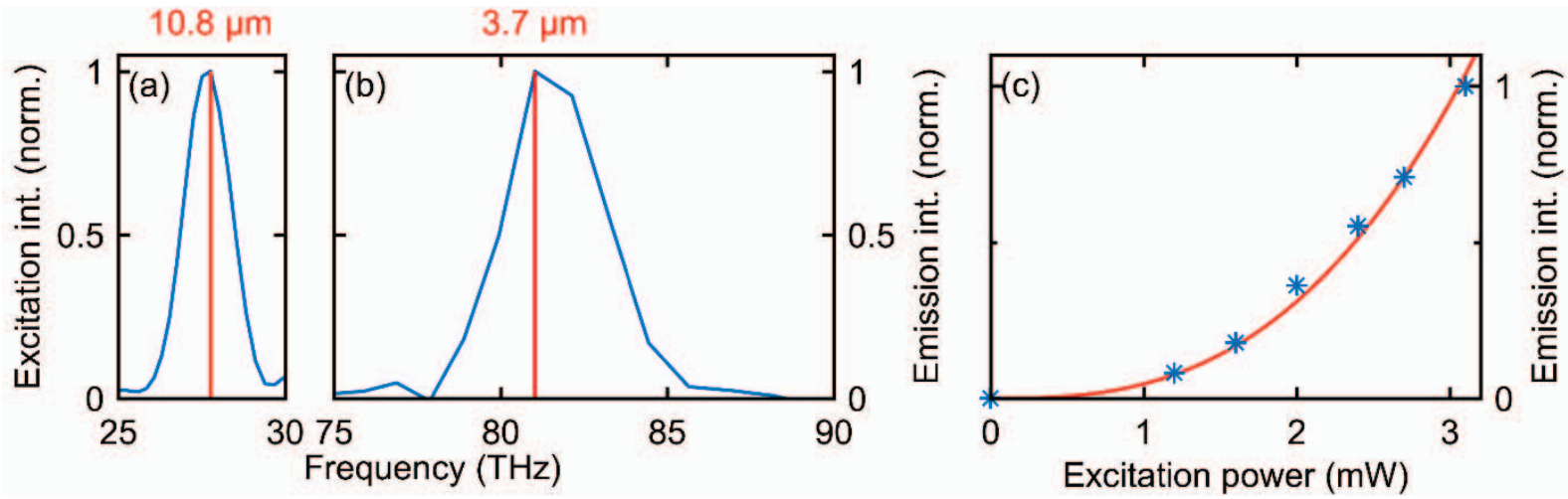

Fig. 2. (a) Normalized excitation and (b) THG emission intensity spectra from a germanium nanoantenna; (c) power dependence of the nonlinear emission of a single Ge antenna (blue: measurement, red: fitting curve $\sim \mathrm{x}^{2.8}$ ).

In conclusion, we demonstrate third-harmonic generation from heavily doped Ge structures illuminated by intense excitation with femtosecond pulses owing to the plasmonic enhancement occurring in the near-field. Such nonlinear optical antennas are sources of ultrashort radiation constrained below the diffraction limit. This work opens interesting perspectives for experiments targeting molecular resonances or other isolated quantum systems in the MIR frequency range.

This research has received funding from the European Union's Seventh Framework Programme (grant agreement $\mathrm{n}^{\circ} 613055$ ) and from the Deutsche Forschungsgemeinschaft (Emmy Noether Program BR 5030/1-1).

[1] L. Baldassarre, E. Sakat, J. Frigerio, A. Samarelli, K. Gallacher, E. Calandrini, G. Isella, D. J. Paul, M. Ortolani, and P. Biagioni, "Midinfrared Plasmon-Enhanced Spectroscopy with Germanium Antennas on Silicon Substrates", Nano Lett. 15, 7225-7231 (2015).

[2] M. P. Fischer, C. Schmidt, E. Sakat, J. Stock, A. Samarelli, J. Frigerio, M. Ortolani, D. J. Paul, G. Isella, A. Leitenstorfer, P. Biagioni, and D. Brida, "Optical activation of germanium plasmonic antennas in the midinfrared", Phys. Rev. Lett. 117, 47401 (2016).

[3] N. K. Hon, R. Soref, and B. Jalali, "The third-order nonlinear optical coefficients of $\mathrm{Si}, \mathrm{Ge}$, and $\mathrm{Si}_{1_{-x}} \mathrm{Ge}_{\mathrm{x}}$ in the midwave and longwave infrared", J. Appl. Phys. 110, 11301 (2011).

[4] T. Hanke, J. Cesar, V. Knittel, A. Trügler, U. Hohenester, A. Leitenstorfer, and R. Bratschitsch, "Tailoring spatiotemporal light confinement in single plasmonic nanoantennas", Nano Lett. 12, 992 (2012).

[5] J. Frigerio, A. Ballabio, G. Isella, E. Sakat, P. Biagioni, M. Bollani, E. Napolitani, C. Manganelli, M. Virgilio, A. Grupp, M. P. Fischer, D. Brida, K. Gallacher, D. J. Paul, L. Baldassarre, P. Calvani, V. Giliberti, A. Nucara, and M. Ortolani, "Tunability and Losses of Mid-infrared Plasmonics in Heavily Doped Germanium Thin Films”, Phys. Rev. B 94, 85202 (2016). 